\title{
Icodextrin and spurious hyperglycemia in peritoneal dialysis patients: a hospital-wide attempt to prevent such errors
}

\author{
Yusuke Asakura $^{1}$ and Keiko Kimura ${ }^{2}$ \\ Departments of ${ }^{1}$ Anesthesiology, ${ }^{2}$ Nephrology, Nagoya Kyoritsu Hospital, Aichi, Japan
}

In peritoneal dialysis (PD), a dextrose-containing solution is infused into the peritoneal cavity, and toxic materials are removed by ultrafiltration and diffusive clearance through the peritoneal membrane. PD is used in $2.9 \%$ of the approximately 320,000 patients regularly undergoing any form of hemodialysis in Japan. Recently, a non-absorbable carbohydrate (icodextrin; Extraneal $^{\mathrm{TM}}$; Baxter Healthcare, Chicago, IL, USA) has been introduced for $\mathrm{PD}$, and more efficient ultrafiltration has been reported. Although icodextrin is usually considered the last step for PD patients with a long dwell, the prevalence of icodextrin use remains unclear. Importantly, serious spurious hyperglycemia perioperatively has been reported with icodextrin [1-3].

In our facility, 45 patients regularly undergo PD in an outpatient setting. Of these, $14(31 \%)$ patients use icodextrin as the dialysate.

Classically, glucose oxidase is used in bedside glucometers. A major drawback is its cross-reactivity with dioxygen, resulting in an underestimation of the blood glucose. As an alternative, soluble quinoprotein glucose dehydrogenase (GDH) with the co-factor pyrroloquinoline quinone (sPQQ-GDH) was developed. This enables measurements without any interference from dioxygen, although low substrate specificity and cross-reactivity with maltose, a metabolite of icodextrin, have been reported. A group of Japanese researchers discovered a novel thermostable heme-containing flavin adenine dinucleotide (FAD)-GDH with high substrate specificity [4]. A FAD-GDH-dependent bedside glucometer is now available commercially, and the list of glucometers is available from the website of Baxter. In May 2015, all of the glucometers in our hospital were replaced and no problems have occurred since then. The cost of the tips for glucometer does not substantially differ from those of sPQQ-GDH glucometer.

\section{References}

1. Kroll HR, Maher TR. Significant hypoglycemia secondary to icodextrin peritoneal dialysate in a diabetic patient. Anesth Analg 2007; 104: $1473-4$.

2. Riley SG, Chess J, Donovan KL, Williams JD. Spurious hyperglycaemia and icodextrin in peritoneal dialysis fluid. BMJ 2003; 327: 608-9.

3. Perera NJ, Stewart PM, Williams PF, Chua EL, Yue DK, Twigg SM. The danger of using inappropriate point-of-care glucose meters in patients on icodextrin dialysis. Diabet Med 2011;28: 1272-6.

4. Tsujimura S, Kojima S, Kano K, Ikeda T, Sato M, Sanada H, et al. Novel FAD-dependent glucose dehydrogenase for a dioxygen-insensitive glucose biosensor. Biosci Biotechnol Biochem 2006; 70: 654-9.

Corresponding author: Yusuke Asakura, M.D., Ph.D.

Department of Anesthesiology, Nagoya Kyoritsu Hospital, 1-172, Hokke, Kakagawa-ku, Nagoya, Aichi 454-0933, Japan

Tel: 81-52-362-5151, Fax: 81-52-353-9105, Email: yasakura@kaikou.or.jp

ORCID: https://orcid.org/0000-0003-3966-1757

Received: February 24, 2017. Accepted: March 19, 2017.

Korean J Anesthesiol 2017 August 70(4): 479

https://doi.org/10.4097/kjae.2017.70.4.479

(c) This is an open-access article distributed under the terms of the Creative Commons Attribution Non-Commercial License (http://creativecommons.org/ licenses/by-nc/4.0/), which permits unrestricted non-commercial use, distribution, and reproduction in any medium, provided the original work is properly cited. 